María del Pilar Unda Bernal*

Aroldo Guardiola IBARRA*

Soraya CONSTÁN MEDERO***

OfElia BerRío MORELO*****

MARGARITA MARTíNEZ CAMACHO*****

\title{
PEDAGOGÍA, INTERCULTURALIDAD Y FORMACIÓN DE MAESTROS:ESCUELA NORMAL SUPERIOR INDÍGENA DE URIBIA (LA GUAJIRA) ${ }^{\text {watume }}$
}

\section{Resumen}

Este artículo resume la investigación realizada sobre un conjunto de prácticas pedagógicas que se realizan actualmente en la Escuela Normal Superior Indígena de Uribia, reconocidas y nombradas como pedagogía de la afirmación cultural por la Expedición Pedagógica. El estudio identifica momentos, características, factores y acontecimientos sociales y políticos relacionados con una forma de hacer escuela que rompe con los propósitos de invisibilización y homogeneización de las culturas, se orienta a la valoración y fortalecimiento de la etnia wayúu y evidencia trazos de un trabajo en perspectiva de interculturalidad.

Palabras clave: Prácticas pedagógicas, diversidad cultural, multiculturalidad, interculturalidad.

* Candidata a Doctora en el Programa de Cultura y Educación en América Latina, de la Escuela Latinoamericana de Postgrados y Políticas Públicas, Elap, de la Universidad de Artes y Ciencias Sociales, Santiago de Chile. Profesora de la Universidad Pedagógica Nacional e integrante del equipo de trabajo coordinador del Movimiento Expedición Pedagógica.Correo electrónico: pilunda@gmail.com

** Magíster en Estudios Político-económicos, Especialista en Planeación Territorial, Licenciado en Lenguas Modernas, Coordinador de la Ruta Afrocaribe de la Expedición Pedagógica, integrante del equipo de coordinación del Movimiento Expedición Pedagógica del Caribe. Correo electrónico: nodocaribe@yahoo.com

*** Licenciada en Etnoeducación y Proyectos Sociales, Especialista en Pedagogía de la Lengua y la Literatura, Maestra de Cultura y Lengua en el Ciclo Complementario, Maestra anfitriona de la Ruta Escuelas Normales Región Caribe Expedición Pedagógica.Correo electrónico: ofebemo@gmail.com

**** Licenciada en Ciencias Naturales, Especialista en Evaluación Escolar, Profesora de Investigación y Coordinadora del Ciclo Complementario de la Ensui, maestra, viajera y anfitriona de la Ruta Escuelas Normales Expedición Pedagógica.Correo electrónico: ofebemo@ gmail.com

${ }^{* * \star * \star}$ Licenciada en Lenguas Modernas, Especialización en Metodología de la enseñanza de la lengua y literatura, Especialización en Orientación Educativa y Desarrollo Humano. Maestra de Teoría Lingüística y de Didáctica de la Lengua en el Ciclo Complementario de la Escuela Normal Superior Indígena de Uribia, expedicionaria anfitriona.Correo electrónico: ofebemo@gmail.com

${ }^{* * * * * \star}$ Este artículo es una síntesis de la investigación financiada por el Fondo Concursable para el Desarrollo de Proyectos de Innovación e Investigación en Educación Inclusiva y Convivencia Democrática, organizado por la Red Innovemos de Unesco, Chile. Una versión ampliada fue publicada en Orealc-Unesco (2008). Educación y diversidad cultural. Lecciones desde la práctica innovadora en América Latina. Santiago de Chile. http://www.redinnovemos.org

Articulo recibido el 16 de abril de 2009 y aprobado el 24 de junio de 2009

Pedagogía y Saberes N.ํㄹㄹ 28. Universidad Pedagógica Nacional. Facultad de Educación, 2008, pp. 113-120 


\title{
PEDAGOGIA, INTERCULTURALDAD E FORMAÇÃO DE MAESTROS: ESCOLA NORMAL SUPERIOR INDÍGENA DE URIBIA (LA GUAJIRA)
}

\section{Resumo}

Neste artigo apresenta-se o resumo da investigação realizada num conjunto de práticas pedagógicas que se realizam na atualidade em a Escola Normal Superior Indígena de Uribia. Ditas praticas reconhecidas como Pedagogia da Afirmação Cultural pela Expedição Pedagógica. O estudo identifica momentos, características, fatores e acontecimentos sociais e políticos relacionados com uma forma de fazer escola que rompe com os propósitos de homogeneização das culturas, visando orientar-se na valoração e fortalecimento da etnia wayúu- $O$ trabalho evidencia rasgos em perspectiva de interculturalidad.

Palabras Chave: Práticas pedagógicas, diversidade cultural, multiculturalidad, interculturalidad.

\section{PEDAGOGY, INTERCULTURAL AND TEACHER EDUCATION: INDIGENOUS PRIMARY SCHOOL TEACHERS SCHOOL OF URIBIA}

\begin{abstract}
This article summarizes a research carried out upon a set of pedagogical practices that are carried out at present in the INDIGENOUS PRIMARY SCHOOL TEACHERS SCHOOL OF URIBIA (La Guajira), which has been recognized and named as Pedagogy of Cultural Affirmation by the Pedagogical Expedition. The study identifies moments, characteristics, factors and social and political events related to a form of 'making school' that breaks off the purposes of no visibility and homogenization of the cultures. It has been directed towards the appraisal and strengthening of the Wayúu ethnic group and gives evidence of a work in perspective of intercultural.
\end{abstract}

Key words: Pedagogical practices, cultural diversity, multiculturalism, intercultural.

Durante los últimos diez años, se adelanta en Colombia la Expedición Pedagógica Nacional, un movimiento social y educativo de maestras y maestros que recorren pueblos y ciudades para el encuentro y el reconocimiento de la diversidad pedagógica existente. La riqueza de saberes y de prácticas, así como el atrevimiento de los maestros que las ponen en marcha, han puesto en evidencia los límites de las aproximaciones que se han hecho al tema de la pedagogía como campo de saber.

Se ha puesto en escena un maestro que se reconoce productor de saber, rompiendo así con las relaciones jerárquicas, de dependencia y de subordinación que le han negado su condición de sujeto y que pretenden limitar su papel al de simples consumidores de la producción de conocimientos elaborada en otros lugares.

El movimiento expedicionario se erige como una expresión actual del Movimiento Pedagógico, surgido en Colombia en la década del ochenta. Al definir el viaje como su estrategia básica de contacto con las prácticas de los maestros, al proponer otras formas de interacción desde lo regional y al reconocer la diversidad cultural y pedagógica existente en nuestro país, adquiere una connotación específica y un dinamismo propio.

El viaje realizado en 2002 a la Escuela Normal Superior Indígena de Uribia ${ }^{1}$, Ensiu, sorprendió a los viajeros de la Ruta de las Normales de la Expedición Pedagógica del Caribe $^{2}$. El recibimiento

1 La Escuela Normal Superior de Uribia es una institución formadora de maestros que articula los niveles educativos preescolar, primaria, secundaria y ciclo complementario. Esta institución escolar congrega la diversidad étnica y cultural existente en el municipio de Uribia y en otras localidades vecinas.

2 Este viaje, en el que participaron maestras y maestros de las Escuelas Normales, incluyó los tres departamentos del antiguo Magdalena Grande: La Guajira, Cesar y Magdalena. de las estudiantes, maestras en formación, ataviadas con sus multicolores mantas guajiras; el rancho Shipia Wayüu, construido a la entrada de la escuela por maestros y estudiantes para visibilizar la cultura material wayüu; los chinchorros en el dormitorio de las internas y el recibimiento con el Himno Nacional de la República de Colombia entonado en wayuunaiki por los estudiantes predecían la riqueza pedagógica que despliega esta escuela formadora de maestros. Pero también produjo un extrañamiento en maestras, maestros y directivas anfitriones, quienes pudieron verse en el espejo de los viajeros, con sus lecturas, preguntas y comentarios, con sus modos de sorprenderse, como la ficha que hacía falta en el rompecabezas, una imagen de sí que hace visible una parte de toda esa riqueza y potencia de sus propias prácticas.

En el encuentro nombramos como pedagogía de la afirmación cultural a esta forma de hacer pedagogía que no aparece en los tratados de pedagogía ni pretende asemejarse a los enfoques 
o "sistemas" en ellos allí reconocidos. La pedagogía, en este caso, como en otras escuelas visitadas por los expedicionarios, no se construye sobre un vacío, sino a ras de tierra, en interacción con los territorios, con las culturas, con las condiciones económicas y sociales de nuestras poblaciones ${ }^{3}$. Al concluir el viaje, un dato desconcierta: no siempre la Escuela Normal ha orientado así sus prácticas, hubo tiempos en los que trataría de ignorar, en el mejor de los casos, o de borrar la cultura wayüu. La Constitución Nacional, expedida en 1991, habría contribuido a producir este viraje. Un importante motivo para la realización de este estudio.

El objetivo de esta investigación apunta a identificar los orígenes, momentos, características, factores o acontecimientos sociales y políticos relacionados con esta práctica pedagógica de la Escuela Normal, como institución formadora de maestros.

\section{NUESTRA RUTA METODOLÓGICA}

Los fundamentos teóricos y metodológicos de esta investigación responden a las conceptualizaciones que ha construido el movimiento expedicionario. Se nutre de las corrientes epistémicos críticas que, articuladas a la investigaciónacción participativa, surgen en el país en los años ochenta, reconocen nuestras condiciones vitales como país tropical, con su diversidad regional, étnica y cultural, toman distancia de los paradigmas eurocéntricos, sin ignorarlos, y reivindican la necesidad de paradigmas que privilegien la búsqueda de una creatividad propia (Fals Borda y Mora-Osejo, s. f.). Resaltar el lugar de los sujetos sociales, de sus saberes y de la experiencia es un asunto que adquiere cada vez mayor importancia, especialmente en relación con los movimientos sociales actuales en América Latina. Esta perspectiva

3 Ha sido precisamente esto lo que ha llevado a proponer una noción como la de geopedagogía, en el marco de la Expedición Pedagógica Nacional. Ver Messina y Quiceno (2002). epistémica redefine el papel del investigador, las relaciones sujeto-objeto de la investigación y reconoce el carácter histórico indeterminado, indefinido, no acabado y relativo del conocimiento.

La Expedición posibilita la participación de los individuos en la elaboración de las experiencias, a partir de sus vivencias en la escuela. Una práctica se convierte en experiencia "sólo cuando aquella es pensada, cuando se reconoce como producto de un saber o de una cultura" (Martínez, Unda y Mejía, 2002, p. 92).

Nuestra ruta se inicia con el reconocimiento y valoración de la diversidad étnica y cultural que existe en Colombia $y$, de manera especial, en el departamento de La Guajira, en cuyo territorio coexisten e interactúan diversos grupos étnicos, destacándose la comunidad indígena wayüu. La metodología implementada se basó en el uso de un instrumento polifónico (diversos formatos para recoger las voces de los actores del proceso), documentación bibliográfica, grupos focales y grupos de discusión entre maestros y maestras, estudiantes, dirigentes educativos y reconocidos líderes de la comunidad wayüu en el municipio de Uribia.

La realización de esta investigación compromete a un equipo de la Expedición Pedagógica Nacional y a maestras de la Escuela Normal Superior Indígena de Uribia, una de las cuales es maestra wayüu. Ello posibilitó la confluencia de distintos saberes, así como una activa y permanente fuente primaria muy valiosa a la hora de interpretar los textos y los registros de los talleres y grupos de discusión, las entrevistas y las observaciones realizadas. Este proceder metodológico tiende puentes entre los saberes más cercanos a la academia universitaria y los saberes pedagógicos que circulan y se expresan en la vida de las escuelas. Busca desestabilizar "los lugares autoritarios de emisión de discurso y de emisión de política y 'recomunicar' la esfera académica, la esfera pública y la esfera política, como se propone en algunos planteamientos sobre la crisis de los intelectuales"4.

Más que contrastar la vida de la escuela con un determinado "marco teórico", se trata de recurrir a las teorías a la manera de caja de herramientas, para atrevernos a pensar aquello que no había sido pensado, para atrevernos a actuar de otras maneras. Es otro ingreso a la producción de conocimiento que se construye desde y no sobre las escuelas y los sujetos de la práctica y de la experiencia.

\section{MULTICULTURALIDAD E INTERCULTURALIDAD: DOS POSTURAS ÉTICAS DIFERENTES}

La escuela, cumpliendo desde el siglo XIX con su función claramente civilizatoria, estuvo orientada a luchar contra la barbarie, bajo el supuesto de que para construir nación era necesario eliminar las diferencias, homogeneizar e integrar a estas poblaciones. La diversidad era un problema que requería ser superado y erradicado. El aporte de esta investigación consiste en documentar otra forma de hacer escuela, un quiebre, una ruptura con las narrativas construidas sobre la misma.

Cuando se hace evidente que la propiedad o la exclusión del capital económico engendra una participación desigual en el capital escolar y, por tanto, en la apropiación de los bienes culturales de los que dispone una sociedad, en América Latina se presenta un decidido interés, particularmente en los sectores medios y populares que antes tenían poco acceso, por mejorar los niveles educacionales y culturales.

La historia de las poblaciones indígenas, como la de las comunidades afrocolombianas, ha sido una historia de invisibilización, exclusión y opresión. Sus demandas de acceso a la educación y

4 Sarlo, B., entrevista realizada por Zerán, Faride, Diario La Época, Santiago de Chile, 15 de junio de 1977. 
de inclusión en el "servicio" educativo forman parte de sus luchas y sus movimientos, apelando en muchos casos a las legislaciones vigentes que ya en el siglo XIX reconocían la igualdad frente a la ley y el derecho a la educación. Inicialmente no ponen en duda la oferta curricular. En un momento en el cual la invisibilidad de la población indígena es muy alta, la posesión de la lengua hegemónica, la lectura y la escritura se convierte en la principal demanda, dado que proporciona los códigos mediante los cuales podían asumir la defensa de sus intereses tanto colectivos como individuales. Sin embargo, esta inclusión parcial desde el dominio lingüístico continúa excluyendo a la población indígena, no tiene en cuenta los saberes, las prácticas, las historias y las tradiciones de las comunidades (López, 2001, p. 8).

Más adelante, en los años setenta, el movimiento indígena en Colombia hace exigencias de una educación "propia", como alternativa frente al mestizaje uniformador. Se ponen en marcha los programas de etnoeducación, dirigidos específicamente a las poblaciones indígenas, centrados en sus territorios, en la adecuación de la escuela a las diferentes culturas, a cada una de ellas de manera particular. El reconocimiento del carácter pluriétnico y multicultural de Colombia en la Constitución Nacional de 1991 marca todo un acontecimiento y queda abierto el debate sobre los diferentes enfoques y comprensiones de las relaciones entre culturas.

La noción de multiculturalidad traduce una realidad de América Latina: nuestras sociedades son, desde siempre, multiculturales, como lo afirma Shmelkels (s. f., p. 4). Sin embargo:

La multiculturalidad puede entender al otro como aquel cuya diferencia tiene que borrar. El sujeto diferente tiene que dejar de ser diferente para poder interactuar con la cultura dominante. No se le deja ser. La multiculturalidad puede, por otro lado, reconocer al otro como diferente, pero apartarlo por ser distinto. Puede dejar al otro ser distinto, pero segregado, en su región de refugio, en su reservación o su ghetto.
La educación multicultural se dirige a "garantizar, de un lado, la reproducción de la multiculturalidad y, de otro, a facilitar un cruce de fronteras ágil y fluido, en beneficio de la sociedad envolvente" (López, 2001, p. 9). Desde algunas posturas, es como si la energía crítica hubiera encontrado una válvula de escape en la pelea por diferencias culturales que dejan intacta la homogeneidad básica del sistema capitalista mundial (Grüner, 2002, pp. 62-90).

La interculturalidad es decisivamente otra postura ética, otra forma de entender la relación entre las culturas, que sabe de la insuficiencia de las luchas particulares si no se tienen en cuenta los procesos más amplios en los cuales se producen las condiciones de inequidad y diferencia. La interculturalidad pasa por el reconocimiento de cada cultura, pero es un asunto que compete al conjunto de la sociedad, puesto que introduce el asunto de las profundas asimetrías existentes.

\section{DE UN ENFOQUE CIVILIZATORIO AL RECONOCIMIENTO Y VIVENCIA DE LA CULTURA WAYÜU}

Podemos entonces distinguir varios momentos en la historia de la práctica pedagógica de la Escuela Normal. Un primer momento, orientado por el propósito, que caracterizó durante casi todo el siglo XX a los estados latinoamericanos, incluido el colombiano, de combatir la barbarie y homogeneizar culturalmente las naciones. La política educativa pretende civilizar o normalizar aquellas poblaciones que se apartan entonces de los parámetros característicos de la ilustración europea. En la Escuela Normal, fundada en 1956, las directivas prohíben el uso del "guajiro", acudiendo para ello a las amenazas de sanción a los estudiantes que infringieran las reglas de comportamiento. Las jóvenes practicantes debían asistir calzadas con zapatos de tacón alto, con vestidos occidentales y bien presentadas. Prohibido terminantemente ataviarse con prendas de la cultura indígena. De esta manera la institución iba encaminada a "alijunizar",

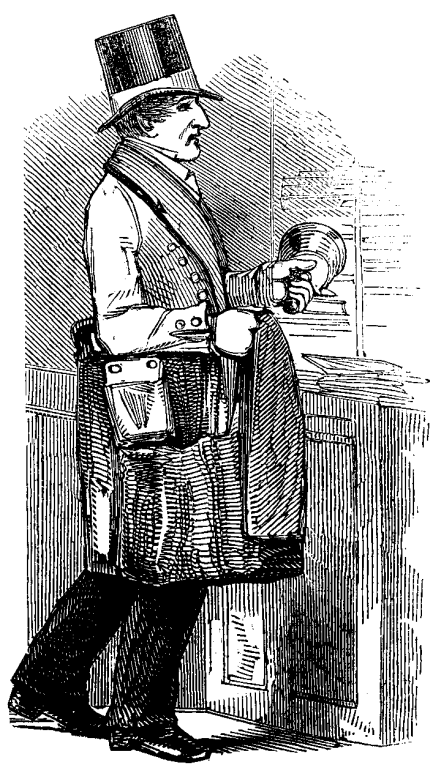

lo que equivale a imponer la cultura occidental agenciada por el Estado mediante sus políticas y programas.

Un segundo momento, cuyas primeras realizaciones devienen de la irrupción del movimiento indígena y del Festival de la Cultura Wayüu a mediados de los años setenta, produce, a partir de 1985 , una primera inflexión, una primera ruptura con las prácticas pedagógicas homogeneizadoras, se comienza a pensar que la cultura wayüu tiene una importancia que "nosotros ignorábamos"5. Esto se ve fortalecido con la llegada a Uribia de académicos venezolanos y colombianos que profundizan en el estudio de la cultura y producen una gramática de la lengua ${ }^{6}$.

$Y$ un tercer momento, que profundiza el anterior, tiene que ver con los postulados

5 Profesor Mario, Proyecto Etnoeducativo Nación Wayuu, p.9.

6 El interés de las universidades colombianas y venezolanas por la cultura wayüu tiene como antecedentes dignos de encomiar los esfuerzos de Gliserio Tomás Pana en el estudio del wayuunaiki, recogidos en un texto publicado en Venezuela, titulado Etimología de la cultura wayüu y el trabajo de Antonio Joaquín López titulado Dolores de una raza. 
incluyentes de la Constitución Política de 1991, que incidió de manera significativa sobre la percepción y la valoración de las comunidades indígenas y fue decisivo, además, para profundizar los procesos pedagógicos actuales. La Constitución, redactada por la Asamblea Nacional Constituyente $^{7}$, reconoce por primera vez la diversidad étnica y cultural de la nación colombiana y derechos tan importantes como la propiedad colectiva de los territorios indígenas, el ejercicio de funciones jurisdiccionales por parte de las autoridades propias en su ámbito territorial y los gobiernos autónomos. La percepción sobre la situación de desventaja económica y social de las comunidades cambia. La Escuela Normal, que anteriormente se veía presionada porque las comunidades buscaban que sus hijos e hijas no fueran educados como wayüu para evitar la discriminación y la exclusión y así tener acceso a mejores oportunidades y condiciones de vida, ahora se encuentra con una población que reclama una educación que les permita reconocerse y afirmarse como tal.

En consonancia con el mandato constitucional, la Ley General de Educación, LGE, reglamenta aspectos como la autonomía de planes, programas y proyectos educativos y recoge importantes planteamientos del Movimiento Pedagógico:

En 1989 las prácticas seguían las líneas alemanas, el maestro era un simple ejecutor, no se podía salir ni un paso porque ya tenía problemas. Estábamos en un mundo diferente, estábamos en La Guajira, pero nuestra guía era Bogotá. La reforma curricular es parecida pero con ciertas diferencias, el paradigma de la tecnología educativa. Surge el Movimiento Pedagógico que se irradia en el país y que trae como consecuencia la filosofía

$7 \quad$ Esta asamblea constituyente fue resultado de una amplia movilización social en el país, por parte de estudiantes, movimientos cívicos y regionales, indígenas, campesinos y movimientos de paz, que demandaban del gobierno central la convocatoria a una Asamblea Nacional Constituyente o Asamblea Constitucional. de la LGE y el PEI que propone autonomía, dependiendo del contexto. Eso influyó mucho porque las políticas nos tenían maniatados (Profesor Mario, Registro).

Este periodo se prolonga hasta nuestro días, con marcadas tensiones con el proyecto educativo homogeneizador. Se caracteriza porque la práctica pedagógica se piensa y se realiza en interacción con la cultura wayüu, genera aproximaciones, estudios y procesos de reconocimiento y de afirmación cultural y pone en marcha, a la vez, dinámicos procesos interculturales.

\section{PEDAGOGÍA DE LA AFIRMACIÓN CULTURAL}

Los aspectos que se resaltan en este estudio no se encuentran separados, por el contrario, se tejen entre ellos hilos muchas veces invisibles que conectan diferentes actividades y realizaciones, no siempre previstas o pensadas de manera articulada, cuyo eje es la cultura:

[...] La cultura es parte esencial de cada una de las materias y de todo el pensum escolar, no es aislada, es un proceso que va insertado en cada uno de nosotros, puede ser el maestro de español, puede ser el maestro de matemática, puede ser el maestro de educación física, puede ser el maestro de informática, que todos tenemos que tocar a todos nos llega esa cultura. $Y$ a través de eso, vuelvo y te digo, la fortalecemos, la intercambiamos con la cultura occidental, con la cultura wayüu y otras culturas que ha habido en la Escuela Normal, porque en otros años teníamos kogui, teníamos otros grupos indígenas y de ellos cuánto no aprendimos y ellos cuánto no aprendieron de nosotros [...] (Entrevista Amalia Castillo).

Hemos organizado su presentación en torno a los siguientes núcleos:

\section{Wayuunaiki y bilingüismo}

Se entiende que la lengua está ligada a la vivencia y la comprensión de la cultura indígena. Por ello, un aspecto esencial de la práctica pedagógica de la Normal es el abordaje de la cultura y la lengua wayüu, el wayuunaiki, en los espacios académicos y en el conjunto de la vida escolar. Una maestra wayüu es la responsable de la enseñanza de la lengua en todos los grupos, desde el primer grado hasta el ciclo complementario:

Aquél que no habla la lengua no sabe de su cultura, [...] está en nuestras cabezas, está en nuestros corazones. Quienes no hablan la lengua no entienden, porque nos viene de adentro, el ombligo es lo que nos cuelga al chinchorro, lo que nos pega en el pensamiento (Zoraya Constan, maestra wayüu, integrante de este equipo de investigación).

La Escuela Normal es lugar de encuentro de maestros y estudiantes wayüu y alijunas. Un alto porcentaje de estudiantes wayüu ha crecido en el seno de hogares conformados por wayüu y alijuna, donde no se habla el wayuunaiki, ésta no ha sido su lengua materna, en muchas familias se subvalora. Los estudiantes reclaman su deseo de aprenderla:

Mi mamá es wayüu pero mi papá no. Mi mamá vivía en una ranchería, cuando salió y se fue a vivir al casco urbano se dejó de practicar el wayuunaiki [...] ahora le reclamo a mi mamá por qué no me enseñó [...] ahora me habla en wayuunaiki8.

La Ensiu conoce bien estas condiciones de la cultura, su propuesta es la de una pedagogía bilingüe e intercultural. Promueve el bilingüismo como vivencia, como cátedra -en la que se estudian las estructuras gramaticales específicas de cada lengua - y como forma de abordar las distintas materias de estudio ${ }^{9}$. Me-

8 Rafael, estudiante del Ciclo Complementario en la Ensiu, Registro grupo discusión entre estudiantes del Ciclo, Uribia, 1 de noviembre de 2007.

9 Durante los grupos de discusión entre los maestros, como en el realizado con los estudiantes del Ciclo Complementario, surgieron diferentes problematicas relacionadas con la enseñanza de la lengua, entre ellas su enseñanza en el nivel preescolar, porque en los actuales momentos en este nivel sólo se imparte 
recen un lugar especial sus obras de teatro, de un hondo contenido cultural y estético, en las cuales los actores (estudiantes) establecen un juego de lenguaje, entrecruzando el wayuunaiki con el español, de manera tal que la obra puede ser entendida en cualquiera de las dos lenguas.

\section{Entrecruce de saberes y senderos: ser wayüu e interculturalidad}

La pedagogía de la afirmación cultural atraviesa la vida de la institución también en las formas de abordar las distintas asignaturas: en la enseñanza de las ciencias naturales se reflexiona sobre el saber ancestral y la ciencia y la tecnología occidental; la clase de cultura material da cuenta también de la literatura y la cosmovisión wayüu. En los proyectos de investigación de los estudiantes del Ciclo Complementario, las diferentes manifestaciones culturales son objeto de la pedagogía: las artesanías, las plantas medicinales, el origen de los clanes, el pastoreo, la pesca, la cerámica, el encierro, los velorios, las huellas, la vivienda, el liderazgo del palabrero.

Los estudiantes de cuarto y quinto de primaria, por ejemplo, desarrollan una actividad de reconocimiento de los usos de las plantas, organizan herbarios que registran la variedad, el uso de las plantas medicinales y la manera de prepararlos. Por su parte, el semillero de investigación, conformado por los estudiantes del Ciclo Complementario, como parte de su formación de maestros, documentan la manera en que los indígenas trabajan el trupillo, el cardón y la producción de cabuya. Y en el componente artístico, la maestra, mediante el tema de la composición, conduce a los jóvenes estudiantes de octavo grado a que realicen, a partir del estudio de las plantas medicinales, trabajos artesanales como la mochila, cuyo proceso de elaboración se describe, se recoge y publica en cartillas pedagógicas.

la enseñanza de la lengua castellana por parte de maestras alijunas.
El estudio de la cosmovisión wayüu es transversal y se realiza desde el grado primero hasta el ciclo complementario. Investigaciones sobre ser wayüu, la manera en que este grupo indígena asume la vida y la muerte. Cómo surgió el Dios de la Lluvia, el Dios del Sol, el Dios de la Luna, los sitios sagrados donde al wayüu que muere se le sepulta por segunda vez para que descanse definitivamente, cinco años después de haber sido sepultado de manera transitoria.

En esta práctica pedagógica de la afirmación cultural, se presenta una estrecha relación entre los contenidos y las maneras de abordarlos. Los objetos de trabajo son tratados de manera interdisciplinar, tanto las áreas, que allí se denominan "componentes", como la vida institucional se conectan con ser wayüu. $Y$ desde allí se abordan, a la manera de entrecruce de saberes y senderos, los aportes de la cultura occidental. Las estudiantes del ciclo complementario aprenden cómo se ha investigado desde la perspectiva de occidente, pero no como forma superior o punto de llegada de la manera de investigar wayüu.

\section{Método de la consulta a los mayores}

La comunidad wayüu tiene una manera particular de aprender y de enseñar: la pregunta y el encuentro con los mayores, observando, oyendo, participando en las actividades. Y ésta es la forma de investigar, de "ir más allá de lo que se puede ver"10, de conseguir que los estudiantes de diferentes grados escolares se acerquen a las prácticas culturales de sus ancestros. Con ello contribuyen a la reconstrucción de aquellas en riesgo de desaparecer, como la forma de fabricar cabuya, para lo cual los estudiantes visitan a una mujer mayor, se extrañan, observan, aprenden. Pero también documentan, escriben, comunican.

10 Palabras de una estudiante del Ciclo Complementario para referirse a la manera de entender la investigación en la Ensui durante el viaje realizado por la Expedición en 2002, notas trabajo de campo.

\section{El arte y la estética en la formación}

Los resultados de las investigaciones realizadas por estudiantes y maestros, mediante el método de la consulta a los mayores, se convierten en herramientas para la creación y recreación estética que, a su vez, interactúa y alimenta los demás componentes y núcleos pedagógicos de la propuesta. Con los estudios realizados por los niños y niñas sobre la cosmovisión, los mitos y las leyendas de la comunidad indígena se han realizado obras como El origen de las deidades wayuu, El origen de la sal o El origen del arcoiris y el color de los pájaros, cuyos títulos son apenas un anuncio del lugar que ocupa la estética en la Escuela Normal11.

La actividad artística abarca también la enseñanza de los instrumentos musicales, las danzas, los tejidos y las manifestaciones folclóricas. Pero no son actividades aisladas, puesto que, para la comunidad wayüu, están profundamente ligadas a su historia, a su cosmovisión y a su religión. En el arte se manifiesta la espiritualidad wayüu, "en los diseños de un chinchorro o de un sombrero, en la yonna, en el canto de un Jaiechi, o en la interpretación de un instrumento musical" (Berrio, Pimienta y Martínez, Margarita, 2006, p. 216).

\section{Producción de materiales sobre la cultura}

Durante el transcurso del trabajo realizado, se producen unos materiales sobre la cultura, que a su vez se convierten en objetos de estudio en la Escuela Normal y aportan a otras actividades, como el Festival de la Cultura Wayüu.

Es el caso de la creación del Shipia Wayüu $^{12}$, en la sede de la institución, basándose en las elaboraciones sobre los contenidos de la cultura material. Su construcción es el resultado de las entrevistas, de los intercambios entre maestros, maestras y estudiantes con

11 Las obras han sido dirigidas por la profesora María Concepción Campo.

12 Vivienda, rancho wayüu. 
los líderes y las autoridades de la etnia wayüu, a partir de la elaboración de proyectos específicos cuyo contenido involucra desde el uso del barro y el yotojoro ${ }^{13}$ para la construcción del rancho, pasando por la variedad de tejidos de los vestidos, la orfebrería para las artesanías, los instrumentos musicales, los símbolos y sus formas de organización en clanes, hasta los productos elaborados con el trupillo, el eucalipto, el cactus y otras plantas como la bija y el dividivi.

También se destaca la construcción del Diccionario Bilingüe, por parte de un grupo de maestras de cultura y lengua de distintas instituciones escolares del municipio de Uribia, con el propósito de fortalecer la lengua y "unificar criterios para su enseñanza", en vista de la diversidad de formas lingüísticas de pronunciaciones y escrituras del wayuunaiki existentes.

\section{Día Étnico y Feria de la Ciencia}

El Día Étnico es una fecha para la celebración, para la fiesta y para la afirmación de la cultura. El dominio del wayuunaiki, la vestimenta y la comida típica, las tradiciones, los ritos y las costumbres de la etnia son los asuntos, los temas y el objeto principal de este acontecimiento escolar. Es una oportunidad para mostrar la transversalidad del estudio de la cultura. Los profesores demuestran sus aportes específicos al abordaje de la cultura en sus trabajos del aula: traducciones del inglés al wayuunaiki, el calculo wayüu, sus formas de contar, la palabra, la organización social, el trabajo colectivo, la historia de este pueblo aborigen. Se destaca también la Feria de la Ciencia, que con el paso de los años varía el foco de su atención: del funcionamiento de los fenómenos naturales, como los volcanes o la creación de una fuente luminosa o la producción de vino de corozo, a relacionar los asuntos de la ciencia con la cultura tradicional.

Se establece un interés simultáneo por el conocimiento científico y el saber

13 Fibra obtenida del cactus disecado. ancestral transmitido mediante la tradición oral en la comunidad indígena. No se presenta, por tanto, una relación de exclusión, de separación, sino de encuentro y coexistencia. Se validan en la Escuela Normal ambas maneras de conocer, de acercamiento e interpretación de la realidad. De hecho, se resalta el saber transmitido por los abuelos de generación en generación, al mismo tiempo que se entra en contacto con los aportes del conocimiento científico, las búsquedas en Internet, la complejización de distintas formas de conocer y visiones de mundo.

La aceptación y el reconocimiento de la existencia de distintas racionalidades que se encuentran en la base del saber tradicional y el conocimiento científico, enmarcado en contextos socioculturales diferentes, posibilita la reflexión en el aula y en la institución sobre las distintas maneras de explicar el mundo. La Feria de las Ciencias se ha convertido entonces en un espacio y una oportunidad para ampliar la mirada sobre las ciencias, no reducida a las ciencias naturales, sino que abarca los temas relacionados con las humanidades y el saber ancestral wayüu.

\section{PARA CERRAR...POR AHORA}

Decíamos, en un artículo anterior, que aquí se desdibujan las fronteras de la escuela, no es fácil distinguir el entre el "adentro" y el "afuera", estas prácticas pedagógicas están inmersas en la cultura wayüu, le aportan y a la vez se constituyen desde y en interacción con ella (Unda y Guardiola, 2003, pp. 58-61).

Esta interacción continúa por medio de las prácticas de las estudiantes del Ciclo Complementario que, como parte de su formación como maestras en la Ensui, hacen presencia constante en rancherías y escuelas del departamento ${ }^{14}$. A

14 En un viaje reciente a la escuela de la Ranchería El Arroyo, durante la preparación de los viajes expedicionarios que se iniciarían a finales de 2007 por los municipios de La Guajira, nos sorprendió la similitud de su enfoque pedagógico partir del reconocimiento de las condiciones sociales y culturales de cada lugar, las estudiantes construyen sus prácticas y producen propuestas pedagógicas que se convierten, a su vez, en acumulado para seguir pensando las prácticas en la Normal y en otras instituciones educativas $^{15}$.

Una vez reflexionada, nombrada y documentada esta práctica como pedagogía de la afirmación cultural en el encuentro de los expedicionarios con maestras y directivas la Escuela Normal Indígena de Uribia, ésta fue reconocida y evaluada por los pares académicos, que, en representación del Ministerio de Educación Nacional y sus planes de acreditación, visitaron a la Escuela Normal y le otorgaron la certificación de calidad y excelencia. Se requirió este proceso de reconocimiento y valoración de las prácticas en marcha y de los saberes que se ponen en juego por parte de maestras, maestros, estudiantes y directivas de la Escuela Normal. Durante el proceso seguido anteriormente se habían estrellado con la exigencia de precisar cuál de los "modelos pedagógicos" sigue la Ensui, como requisito para ser acreditada por parte del MEN. Como resultado, la Ensui, ilntercultural y bilingüe, se había visto

con el que aquí hemos descrito como pedagogía de la afirmación cultural, si bien se abordaban otras problemáticas y temáticas. Las averiguaciones nos llevaron a identificar que algunas de las maestras habían sido estudiantes en la Ensui.

15 A manera de ejemplo, este año el Proyecto Yatawara (contar) permitió construir un modo de enseñar matemáticas que incorpora el saber wayüu. Para ello, las estudiantes, junto con los niños a su cargo, siguieron el procedimiento de consultar a los mayores y de hacer observaciones en el mercado y en las rancherías, lo cual les permitió identificar cómo procede el wayuu para hacer sus cuentas, las unidades de medida que utiliza -el plato, para vender camarones, o el pote, para vender el maíz-y la manera de saber si el número de chivos que vuelve al corral está completo -haciendo un nudo en el tejido por cada animal que sale del corral y desatándolos a medida que ingresan. 
abocada a seleccionar, entre las cuatro opciones planteadas por el Ministerio de Educación, jel énfasis en la lengua castellana! Ha sido un primer resultado que resitúa a la Ensui y la proyecta como una institución formadora de maestros, orientada a la afirmación de la cultura wayüu y a procesos interculturales a lo largo y ancho de la geografía peninsular del departamento de La Guajira.

Una propuesta de esta envergadura sólo es posible por la presencia de maestras y maestros que, en la Escuela Normal Superior Indígena de Uribia, piensan sus prácticas en relación con un profundo compromiso ético y estético con nuestras sociedades y con la cultura:

\section{BIBLIOGRAFÍA}

Berrío, O., Pimienta, V. y Martínez, M. (2006). Pedagogía de la Afirmación Cultural Wayüu. Pedagogía Territorio y Cultura, Expedición Pedagógica Nacional, 8, 209-223

Expedición Pedagógica Nacional. (2005). Con los dedos en la filigrana. Una lectura crítica a los tejidos metodológicos de la Expedición Pedagógica Nacional. Bogotá: Expedición Pedagógica NacionalUniversidad Pedagógica Nacional.

Fals Borda, O. y Mora-Osejo, L. (s. f.). La superación del eurocentrismo. Enriquecimiento del saber sistémico y endógeno sobre nuestro contexto tropical. Extraído desde http://www.revistapolis. cl/7/bals.htm

GRÜNER, E. (2002). ¿Estudios culturales o teoría crítica de la cultura?. En El fin de las pequeñas historias: de los estudios culturales al retorno (imposible) de lo trágico (2002). Buenos Aires: Paidós.

LANDER, E. (ed.). (2000). La colonialidad del saber: euroncentrismo y ciencias sociales. Perspectivas latinoamericanas. Buenos Aires: Consejo Latinoamericano de Ciencias Sociales, Clacso.

LÓPEZ, L. (2001). La cuestión de la interculturalidad y la educación latinoamericana. Séptima Reunión del Comité Regional Intergubernamental del Pro-
Abro las páginas de mi diario motivada por una de las jornadas pedagógicas que desarrollamos aquí, en nuestra Institución, que me hace reflexionar sobre mi quehacer pedagógico y a la vez enamorarme más de mi profesión. Aún no sé bien qué debo escribir ni sé por dónde comenzar; tampoco sé si científicamente tengo mucho conocimiento o no; pero lo que sí estoy segura es que soy maestra por vocación y amo todo lo que hago para y con mis estudiantes (Profesora María Concepción Campo, Diario de Campo, citada en Berrío, Pimienta y Martínez, 2006, p. 219).

Son otras formas de ser maestro, otras formas de hacer escuela, desconocidas

yecto Principal de Educación en América Latina y el Caribe. Unesco.

Martínez, A., Unda, M. y MeJíA, M. (2002). El Itinerario del maestro: de portador a productor de saber pedagógico. En: Veinte años del Movimiento Pedagógico. Entre mitos y realidades. 1982-2002 (pp. 61-94) (2002). Colombia: Cooperativa Editorial Magisterio-Corporación Tercer Milenio

Martínez, M. y Constan, Z. (2004). Rescatando nuestro sentido de identidad a partir de la cultura wayuu como eje de la pedagogía étnica, Trabajo presentado a Colciencias. Mimeo.

MessinA, G. y Quiceno, H. (2002). Evaluación internacional Expedición a la Expedición Pedagógica Nacional, Bogotá, Expedición Pedagógica Nacional-Universidad Pedagógica Nacional-Fundación Restrepo Barco.. .

Montero. M. (1998). Paradigmas, conceptos y relaciones para una nueva era. Cómo pensar las ciencias sociales desde América Latina. Seminario Las Ciencias Económicas y Sociales: Reflexiones de Fin de Siglo. En E. Lander (ed.) (2000), La colonialidad del saber: euroncentrismo y ciencias sociales. Perspectivas latinoamericanas. Buenos Aires: Consejo Latinoamericano de Ciencias Sociales, Clacso. hasta ahora por la investigación educativa, que se obstina en devolver una imagen tan degradada del maestro y por las políticas públicas que, aún hoy, se definen sin su participación.

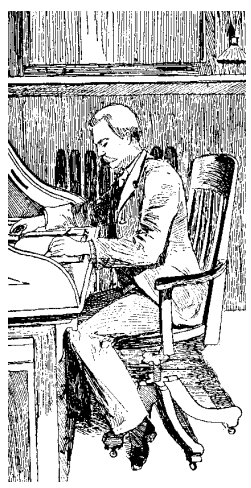

Proyecto Etnoeducativo Nación Wayuu. (2007). Riohacha: Comisión de Redacción.

RAYMONDS, W. (1980). Marxismo y literatura. Barcelona: Ediciones Península.

SARLO, B. (1977, junio 15). En la cultura del zapping. La Época.

SHMELKES, S. (s. f.). Educación intercultural: reflexiones a la luz de experiencias recientes. ponencia presentada en el Congreso La formación de profesores, los desafíos del cambio. International Council on Education for Teaching. Mimeo.

Unda, M. y Guardiola, A. (2003). Pedagogía de la afirmación cultural. Revista Nodos y Nudos, 15, 58-61.

Zambrano, C. (2004). La nación en transición. Dinámicas sociopolíticas del reconocimiento de la diversidad. En: J. Arocha (comp.) (2004), Utopía para los excluidos. El multiculturalismo en Africa y América Latina. Bogotá: Universidad Nacional de Colombia-Unilibro.

ZIZEK, S. (2003). Multiculturalismo o la lógica cultural del capitalismo multinacional. En Estudios culturales, reflexiones sobre el multiculturalismo. (2003). Buenos Aires: Paidós. 